\title{
Relación entre los estilos de vida y autoconcepto en jóvenes universitarios Relationship between lifestyles and self-concept in young university students
}

\author{
*Ángela M aría U rrea-Cuéllar, *Jaime Arenas, *Jonathan Hernández Calle \\ *Institución U niversitaria de Envigado (Colombia)
}

\begin{abstract}
Resumen. 0 bjetivo: relacionar las creencias y las prácticas de los estilos de vida con el autoconcepto en los estudiantes de Psicología de la Institución U niversitaria de Envigado (Colombia). Material y métodos: estudio de corte transversal, con una muestra al eatoria de 165 estudiantes, los instrumentos usados fueron el AF- 5 Cuestionario de Autoconcepto que describe cinco dimensiones: académico/ laboral, emocional, familiar, físico y social, y el Cuestionario de Prácticas y Creencias sobre Estilos deVida, que mide seis dimensiones: condición, actividad física y deporte, recreación y manejo del tiempo libre, Autocuidado y cuidado médico, hábitosalimenticios, consumo de alcohol, tabaco y otras drogas y sueño. Resultados: Losresultados evidencian que la relación entrelas prácticas $(r=-.115)$, las creencias $(r=.026)$ de los estilos de viday el autoconcepto no fue significativa $(p>.05)$, mientrasque la relación entrelas prácticasy las creenciasfue positivay significativa $(r=.697 ; p<.001)$. Conclusión: Se requiere continuar estudiando la relación entre los estilos de vida y el autoconcepto puesto que la evidencia existente de su relación no es concluyente y como resultó, en el presente trabajo, es contradictoria.
\end{abstract}

Palabras clave: estilos de vida, autoconcepto, estudiantes universitarios.

\begin{abstract}
To relate the beliefs and practices about Lifestyles with self-concept in Psychology students at the Envigado University Institution (Colombia). Material and methods: Cross-sectional study, with a random sample of 165 students, the instruments used were theAF5 self-concept questionnaire which described five dimensions: academic / work, emotional, family, physical and social, and the Practices and Beliefs about Lifestyles Q uestionnaire which measures six dimensions: condition, physical activity and sport, recreation and management of free time, self-care and medical care, eating habits, consumption of alcohol, tobacco and other drugs and sleep. Results: The results show that the relationship between practice $(r=-.115)$, belief $(r=.026)$ of lifestyles and self-concept was not significant $(p>.05)$, while the relationship between practices and beliefs was positive and significant $(r=.697 ; p<.001)$. Conclusion: It is required to continue studying the relationship between lifestyles and self-concept since the existing evidence of their relationship is not conclusive, and as it turned out, in the present work, as contradictory.
\end{abstract}

Key words: lifestyles, self-concept, university students.

\section{Introducción}

Los estilos de vida (EV) de acuerdo con lo establecido por laO rganización M undial de laSalud (O MS, 2004) son definidos como «un estado de completo bienestar físico, mental y social» estos entendidos desde los pa trones individuales y las condiciones de vida; además, estudios previos se han interesado en investigar los estilos de vida en población universitaria (Aguirre-Loaiza et al., 2019a; A guirre-Loaiza et al., 2019b; BarbosaGranadosyA guirre-Loaiza2020; Cervanteset al., 2018; Gómez-M azorra et al., 2020; Herazo-Beltran et al., 2020; Rodríguez-Espinosa et al., 2015; Ross-Houle y Q uigg, 2019), gran parte de los estudios indicaron que los estudiantes tienen poco conocimiento, adherencia, motivadores o creencias en torno a los estilos, y que

Fecha recepción: 20-07-20. Fecha de aceptación: 24-12-20

Ángela M aría U rrea Cuéllar

amurrea@ correo.iue.edu.co existe la necesidad de creación de programas que fomenten los estilos de vida en la educación superior; aunque larevisión de literaturade Sánchez-O jeday de Luna Bertos (2015) en la cual muestran que los jóvenes universitarios indican creencias funcionales sobre los estilos de vida pero al mismo tiempo reportan prácticas no saludables.

Por otra parte, Páez y Castaño (2010) y Esteves et al. (2017) exploran los componentes de los estilos de vida, entre ellos los niveles de actividad física en rela ción con los estilos de vida, dejando en evidencia factores de riesgo con respecto a la salud de esta población, por ejemplo: el alto consumo de alcohol, cigarrillo, y poca protección para enfermedades detrasmisión sexual y embarazo, las cuales son consideradas conductas de riesgo. 0 tras investigaciones en Colombia (GómezA costa, 2018) estudiaron factores predictores de los estilos de vida y concluyen que existe poco conocimiento de estos, por lo cual, se debe de trabajar desde las Instituciones de Educación Superior en el mejoramien- 
to de estos factores, pero al mismo tiempo en el contexto universitario se reportan beneficios de un estilo saludable en torno a la práctica sistemática de ejercicio físico en el reconocimiento emocional (Aguirre-Loaiza et al. , 2019a), funcionesneurocognitivas (A guirre-Loaiza et al., 2019b) y calidad de vida (Barbosa-Granados y Aguirre-Loaiza 2020). Así mismo, Herazo-Beltran et al. (2020) describen aspectos psicológicos y sociodemográficos que pueden explicar los estilos de vida que llevan los universitarios, y que direccionan programas de promoción.

En el desarrollo de los estilos de vida, se han identificado diversos factores entre ellos hay uno que se puede ver afectado por las conductas de riesgo y es el autoconcepto, el cual es definido por Harter $(1990,2012)$ como las percepciones que tiene el individuo sobre sí mismo, así mismo A rántzazu et al. (2006) lo han definido como el grado de complacencia con uno mismo y con la vida en general, por su parte Marsh (2001) y Cardenal y Fierro (2003) tienen en cuenta las diferentes dimensiones de la vida como son: la emocional, física, social, laboral y académica. Respecto a este temática se encuentran diversos estudios que han evaluado el autoconcepto en los universitarios, entre estos el de Pinilla-Sepúlveda et al. (2014) en el cual muestran que los estudiantes tienen un buen nivel de autoconcepto, aunque resalta que el físico es mayor en los últimos semestre de la carrera y el emocional es el más bajo; a su vez en la investigación hecha por Veliz-Burgos y U rquijo (2012) evidencian diferencias por sexo, entre las más relevantes es que los hombres tienen mayor autoconcepto emocional y físico, así mismo indican que cuanto más años se tenga se mejoran los niveles en el autoconcepto académico, emocional, familiar y físico. 0 tros estudios reportan una relación positiva del autoconcepto con la calidad de vida (Huebner et al., 2004, Xenakis y Goldberg, 2010) y el rendimiento aca démico (López-Barajas et al., 2010; Cachón-Zagalaz et al., 2015). Así mismo, existen estudios que comparan (O Imedilla et al., 2016) a estudiantes deportista y no deportista en torno al autoconcepto, identificando que los estudiantes deportistas tiene mayores niveles de autoconcepto físico y emocional, en contraste al grupo no deportistas; igualmente, O netti-O netti et al ., (2019) compararon estudiantes de España y Portugal, reportando diferencias en el autoconcepto académico, emocional, físico y vigoroso, siendo los primeros los que tiene autoconceptos moderados.

Por último, se retoma la relación de los estilos de vida y autoconcepto en el estudio deAlves et al., (2017), identificando que son más los jóvenes que adoptan comportamientos saludables frente a los que deciden asumir conductas de riesgo, así mismo mostraron que los que asumen conductas positivas tienen mayor autoconcepto académico. En otro estudio, se indago la moderación del género en los hábitos de vida saludable, los cuales son mejor explicados por un adecuado autoconcepto físico (Guedea-Delgado et al., 2017). Del panorama anterior de antecedentes, se indica que la literatura científica publicada hasta el momento no ha especificado predictores en torno a la práctica y creencias de los estilos de vida sobre diferentes variables del autoconcepto en estudiantes del contexto universitario; de esta forma, conocer el comportamiento entre los estilos de vida y autoconcepto permitirá a las instituciones de educación superior conservar evidencias para orientar planes de acción del área de bienestar estudiantil en relación a directrices de prevención y promoción en salud mental. Por lo anterior, el objetivo del presente estudio es relacionar las dimensiones de los hábitos de vida saludable y del autoconcepto de los estudiantes de Psicología de la Institución Universitaria de Envigado.

\section{Método}

\section{Diseño y participantes}

La población de referencia del estudio fueron 362 estudiantes de segundo, quinto y décimo semestre de la Institución Universitaria de Envigado (Antioquia-Colombia) matriculadosen el segundo periodo del año 2018 en el programa de psicología. Con apoyo del Software Epidat 4.0 se calculó el tamaño de la muestra utilizando un nivel de confianza del $90 \%$, una proporción esperada del $50 \%$ y una precisión absoluta del $5 \%$, para un tamaño total de la muestra de 155 estudiantes. Para la selección de los participantes se realizó un muestreo probabilístico estratificado proporcionado, con el fin de conservar en las muestras por semestre las mismas proporciones encontradas en la población, los estudiantes seleccionados en el muestreo final participaron de ma nera voluntaria. Se utilizó el mismo Software para la selección aleatoria de los estudiantes a evaluar, la muestra total final fue de 165 estudiantes (81\% mujeres), se incrementó el tamaño de la muestra para controlar posibles pérdidas, observándose en el quinto semestre, tal como se expone en la tablal.

El rango de edad osciló entre los 18 y 49 años (La $\left.M_{\text {edda }}=24.3, D E=6.4\right)$, mujeres $n=133,80.6 \%$, hombres $n=32,19.4 \%$. 


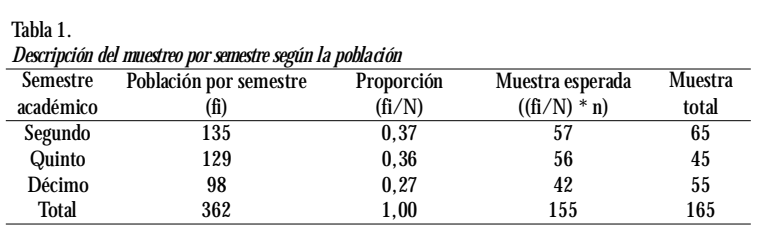

\section{Instrumentos}

Cuestionario de prácticas y creencias sobre estilos de vida

Al eval uar las prácticas y creencias relacionadas con el estilo de vida saludable se administró el cuestionario de Prácticas y Creencias sobre Estilos deVida (Arrivillaga et al. , 2002), este instrumento contiene 116 ítems y dos variables latentes 0 dimensiones: Prácticas y Creencias distribuidas en seis subdimensiones, en Práctica son las siguientes: Condición, actividad físicay deporte (PAF $=6$ ítems), Recreación y manejo del tiempo libre ( $\mathrm{POCIO}=5$ ítems), Autocuidado y cuidado médico (PCUI=21 ítems), Hábitos alimenticios (PALI=17 ítems), Consumo de alcohol, tabaco y otras drogas (PSPA $=10$ ítems) y Sueño (PSUEÑ $0=10$ ítems) y en Creencia: Condición, actividad física y deporte (CAF $=5$ ítems), Recreación y manejo del tiempo libre ( $\mathrm{COClO}=4$ ítems), Autocuidado y cuidado médico (CCUI=13 ítems), Hábitos alimenticios (CALI=7 ítems), Consumo de alcohol, tabaco y otras drogas (CSPA $=11$ ítems) y Sueño (CSUEÑ $0=7$ ítems), el instrumento tiene un formato de respuesta en escala tipo Likert que evalúa la frecuencia del comportamiento mediante cuatro opciones ( $1=$ nunca, $2=$ algunas ve ces, $3=$ frecuentemente y ( $4=$ siempre). Por otra parte, en el presente estudio se calculó la fiabilidad a través del coeficiente 0 mega de McDonald en cada variable latente: (Prácticas sobre Estilos de Vida, ù=.85; Creencias sobre Estilos de Vida, ù=.73; global, ù=.86), también demostró validez de contenido por juicio de expertos (Arrivillaga et al., 2002).

\section{Autoconcepto Forma 5 (AF-5)}

Se aplicó el cuestionario AF- 5 o Autoconcepto Forma 5 (García y Musitu, 2014), este contiene un total de 30 ítems y cinco variables latentes 0 dimensiones: Autoconcepto A cadémico (AA $=6$ ítems), Autoconcepto Social (AS=6 ítems), Autoconcepto Emocional $(A E=6$ ítems), Autoconcepto Familiar ( $\mathrm{AF}=6$ ítems) y Autoconcepto Físico (AFI=6 ítems), se resalta que los ítems 4, 12, 14 y 22 se invierten las respuestas; además, el AF- 5 contiene un formato de respuesta de 99 alternativas de respuestas que evalúa el grado de acuerdo en un continuo de 1 a 99, mediante una escala de actitud tipo Likert ( $1=$ en total desacuerdo hasta $99=$ total mente deacuer- do). El cuestionario ha mostrado utilidad en personas con un rango de edad entre 10 y 62 años (García y M usitu, 2014). Por otra parte, el AF-5 indicó valores de fiabilidad através del coeficientealfa de Cronbach: (AA, $a=.88 ; A S, a=.70 ; A E, a=.73 ; A F, a=.77 ; A F I, a=.74 ;$ global, $a=.82$ ), también demostró validez de constructo en las características psicológicas mediante validez factorial, con una varianza explicada del $51 \%$ a partir de cinco factores (García y Musitu, 2014). Por último, se diseñó un autoinforme ad hoc para recolectar variables sociodemográficas(sexo, edad, estrato socioeconómico, estado civil) y académicas (semestre, promedio académico).

\section{Procedimiento y consideraciones éticas}

Se tuvieron dos momentos principales. La primera, correspondió al contacto con directivas académicas (Decano, director de programa), profesores y estudiantes en segundo, quinto y décimo semestre. Se socializo el objetivo del estudio y las implicaciones que conlleva su participación. La segunda, se ejecutó entre el mes de octubre de 2018 hastajunio de 2019, en estase administró la escala de felicidad, de Goldberg, AF-5 y autoinforme ad hoc, previamente avalado por el consentimiento informado. Por otra parte, la recolección de información se apoyó con la jurisprudencia nacional que regulalainvestigación en Colombia (Colpsic, 2012), específicamente las directrices del capítulo VI artículos 45, 46, 47 y 48. Además, se explicó a los participantes el mínimo riesgo de la investigación descrito en el artículo 11 categoría b resolución 8430 según el Ministerio de Salud y Protección Social de Colombia (Ministerio de Salud - República de Colombia, 1993). Por último, el proyecto fue evaluado y aprobado por el Comité de Ética de la Institución U niversitaria de Envigado (Código 00-122, Acta 11de 2018).

\section{Análisis de datos}

El análisis de datos se realizó en cuatro fases. En la primera, se diseñó una matriz de datos en Excel para ordenar y agrupar la información, en esta se realizó un análisis exploratorio de los datos para identificar y tra tar valores ausentes, atípicos y extremos a partir de recomendacionestécnicas(Aldás\& U riel, 2017; RiveroRodríguez, 2011), por tanto a valores ausentes en una escal a ordinal se imputó la moda a múltiples casos identificados. En la segunda, se redujo el número de varia bles observables (dimensiones) con el Análisis Factorial Exploratorio (AFE) realizado a través del software SPSS versión 22, implementando las pruebas Kaiser-M eyer- 
O lkin (KMO), esfericidad de Bartlett, procedimiento de rotación: Promax, y método de extracción: máxima verosimilitud (Hair, Hult, Ringle, \& Sarstedt, 2017). En la tercera, se utilizó el Análisis Factorial Confirma torio (AFC) en el software AMOS versión 25 , con el objetivo de determinar el número de variables latentes (factores) y observables que se incluyen en la metodología de los M odelos de Ecuaciones Estructurales de tipo reflectivo. En la cuarta fase, luego de haber determina do que variables latentes y observables soportan el AFE yAFC se realizó el análisis de fiabilidad compuesta, va lidez convergente y discriminante del modelo de medida final. Por último, se diseñó análisis de senderos para representar tres variables latentes del modelo inicial (figura 2) y final (figura 3) con sus respectivas medidas de ajuste en cada modelo: Índice de Ajuste Comparativo (CFI), Índice Ajustado de Bonda del Ajsute (AGFI), Error Medio Cuadrático de Aproximación (RMSEA) y PCLOSE.

\section{Resultados}

En la tabla 2 se describe el comportamiento a nivel descriptivo de las dimensionesdel auto-concepto de los estudiantes, la dimensión de mayor puntaje es el auto-concepto académico ( $M=79.85, D E=23.13)$ mientras que la menor puntuación fue el autoconcepto social $(M=44.28, D E=28.83)$; por otra parte, las cinco dimensiones del autoconcepto se concentran entre el percentil 44 y 79, lo cual indicaqueel auto-concepto a nivel global en la población estudiantil conserva un nivel medio.

La descripción de los estilos de vida en relación a la práctica indica un comportamiento de mayor predominio en las diferentes dimensiones, a excepción de AF que reporta una práctica inferior $(M=13.4, D E=3.5)$, mientras que la creencia en las dimensiones CUI, ALI y SPA el puntaje es mayor, la dimensión de OCIO $(M=14.1, D E=1.6)$ y SUEÑO $(M=11.3, D E=1.9)$ la creencia fue menor, en último lugar, se identifica una disonancia notable entre práctica y creencia de los estilos de vida en las dimensiones de CUI, ALI y SUEÑO (Figura 1).

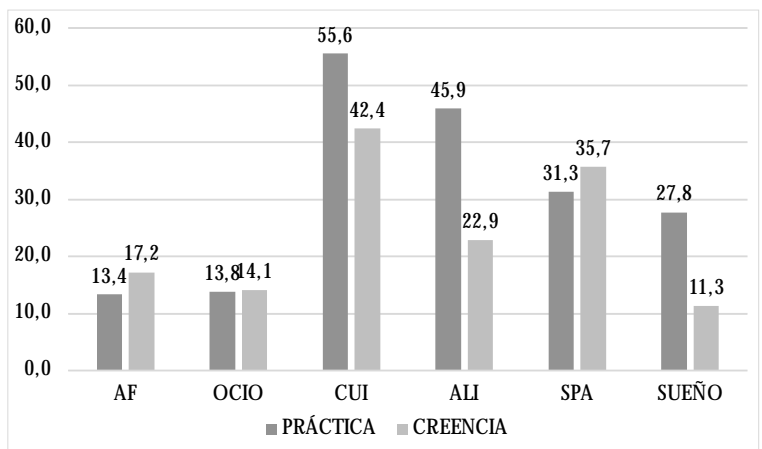

Figura 1. Descripción de las prácticas y creencias de los estilos de vida. $A F=C$ ondición, actividad física y deporte; $\mathrm{OCIO}=$ Recreación y manejo del tiempo libre; $\mathrm{CUI}=$ Autocuidado y cuidado médico; $\mathrm{ALI}=\mathrm{Habitos}$ alimenticios; $\mathrm{SPA}=$ Consumo de alcohol, tabaco y otras drogas; SUEÑO $=$ Sueño.
Tabla 2.

Estadísticos descriptivos de las dimensiones del autoconcepto

\begin{tabular}{cccccc}
\hline \multicolumn{7}{c}{ Dimensiones del autoconcepto AF-5 } \\
\hline Descriptivos & Académico & Social & Emocional & Familiar & Físico \\
\hline Media & 79.85 & 44.28 & 62.63 & 48.07 & 76.78 \\
DE & 23.13 & 28.83 & 32.31 & 32.03 & 25.91 \\
\hline Nota: Ios valores descritos están transformados en puntuación percentil.
\end{tabular}

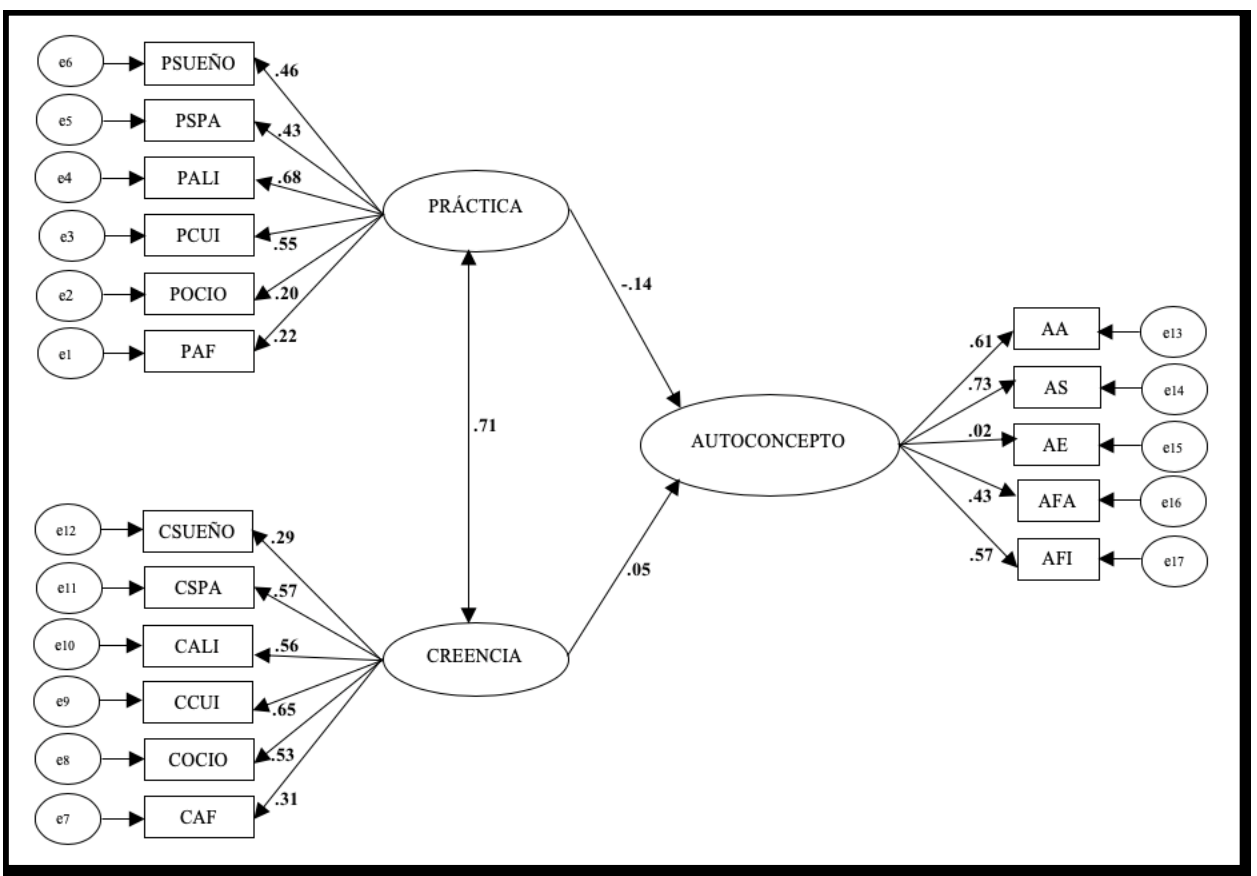

Figura 2: Modelo hipotetizado entre estilos de vida y autoconcepto mediante diagrama de ruta. Práctica: $\mathrm{PAF}=$ Condición, actividad física y deporte; $\mathrm{PO} \mathrm{CIO}=$ Recreación y manejo del tiempo libre; PCUI=Autocuidado y cuidado médico; PALI=Habitos alimenticios; PSPA=Consumo de alcohol, tabaco y otras drogas; PSUEÑ $0=$ Sueño; Creencia: $C A F=$ Condición, actividad física y deporte; $C O C I O=$ Recreación y manejo del tiempo libre; CCUI=Autocuidado y cuidado médico; CALI=Habitos alimenticios; CSPA=Consumo de alcohol, tabaco y otras drogas; CSUEÑO=Sueño. $A A=$ Autoconcepto Académico; $A S=$ Autoconcepto Social; $A E=$ Autoconcepto Emocional; $A F=$ Autoconcepto Familiar; $A F=$ Autoconcepto Físico. 
vadas que subyacen de cada variable latente, este primer modelo (ver figura 2) integrado por una muestra de 165 participantes no presenta un ajuste global satisfactorio $\left(x^{2}=287.67 ; g l=116 ; p<.000 ; C F I=.640\right.$; $\mathrm{AGFI}=.782$, RMSEA $=.095 ;$ PCLOSE $=.000$ ), posterior a diferentes modelos estimados se determina que el modelamiento de ecuaciones estructural es que presenta un ajuste global satisfactorio que se observa en la figura 3 con los siguientes valores $\left(x^{2}=65.36 ; g l=51\right.$; $p<.085 ; \mathrm{CFI}=.952 ; \mathrm{AGFI}=.905, \mathrm{RMSEA}=.041 ;$ PCLOSE $=.668) ;$ sin embargo, es importante tener en cuenta que la prueba chi-cuadrada, como índice, no tiene límite superior, por lo que no puede interpretarse de manera estandarizada, además del problema que plantea su sensibilidad al tamaño de la muestra. Por ello, se muestran otros índices de ajuste estandarizados que son menossensibles al tamaño muestral (Hair et al., 2017; Jöreskog, 1977).

En la tabla 3 y figura 3 exhibe los valores estimados de los parámetros del modelo estructural para los estudiantes evaluados en estilos de vida y autoconcepto. De esta manera se observa que la PRÁCTICA influye de forma directa y significativa en sus respectivas variables observadas PCUI $(r=.512)$, PALI $(r=.691)$, PSPA $(r=$ $.486)$, PSUEÑO ( $r=.438)$; por otra parte, la CREEN CIA evidencia relación positiva y significativa en sus

Tabla 3.

\begin{tabular}{|c|c|c|c|c|c|c|c|}
\hline \multicolumn{3}{|c|}{ Cargas estandarizadas model o se M final en los estillos de } & \multicolumn{4}{|c|}{ PR } & PER \\
\hline \multicolumn{3}{|c|}{ Relación entre variables } & Estimaciones & $\mathrm{EE}$ & $\mathrm{RC}$ & $p$ & Estimaciones \\
\hline AUTOCONCEPTO & $<\cdots$ & PRÁCTICA & -.021 & .037 & -.559 & .576 & -.115 \\
\hline AUTO CONCEPTO & $<\ldots$ & CREENCIA & .007 & .056 & .129 & .897 & .026 \\
\hline PRÁCTICA & $<-->$ & CREENCIA & .214 & .072 & 2.953 & $* * *$ & .697 \\
\hline PCUI & $<--$ & PRÁCTICA & 1.000 & - & 0 & - & .512 \\
\hline PALI & $<--$ & PRÁCTICA & 1.171 & .248 & 4.725 & *** & .691 \\
\hline PSPA & $<--$ & PRÁCTICA & .393 & .096 & 4.113 & *** & .486 \\
\hline PSUEÑO & $<--$ & PRÁCTICA & .414 & .108 & 3.851 & $* * *$ & .438 \\
\hline CCUI & $<-\cdots$ & CREENCIA & 1000 & - & - & - & .624 \\
\hline CALI & $<\cdots$ & CREENCIA & .636 & .115 & 5.507 & $* * *$ & .627 \\
\hline CSPA & $<--$ & CREENCIA & .978 & .18 & 5.428 & $* * *$ & .610 \\
\hline $\mathrm{COClO}$ & $<--$ & CREENCIA & .302 & .065 & 4.667 & $* * *$ & .486 \\
\hline AA & $<--$ & AUTOCONCEPTO & 1.000 & - & - & - & .612 \\
\hline AS & $<--$ & AUTO CONCEPTO & 1.577 & .299 & 5.276 & $* * *$ & .735 \\
\hline AFA & $<--$ & AUTOCONCEPTO & .965 & .232 & 4.162 & $* * *$ & .431 \\
\hline $\mathrm{AFI}$ & $<\cdots$ & AUTO CONCEPTO & 1.435 & .281 & 5.098 & $* * *$ & .575 \\
\hline
\end{tabular}

respectivas variables obser vadas CCUI $(r=.624)$, CALI $(r=.627)$, CSPA $(r=.610)$, COCIO $(r=.486)$.

Igualmente, la variable latente AUTO CO NCEPTO se relaciona de manera positiva y significativa con las diferentes variables observadas de su misma naturaleza teóricaAA $(r=.612)$, AS $(r=.735)$, AFA $(r=.431)$, AFI $(r=.575)$. En cambio, la PRÁCTICA $(r=-.115)$ y CREENCIA ( $r=.026)$ de los estilos de vida no influyen sobre el AUTOCONCEPTO, mientras que existe una relación directa y significativa entre la PRÁCTICA y CREENCIA ( $r=.697)$ de los estilos de vida.

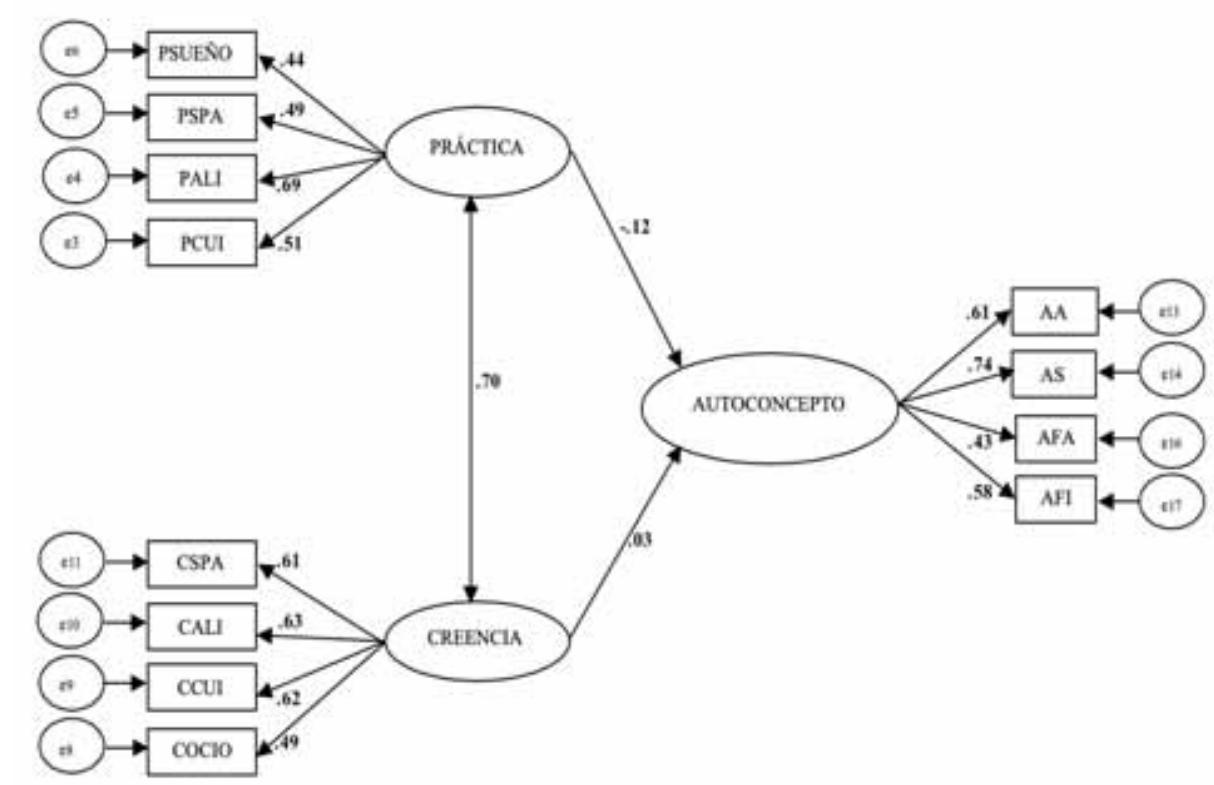

Figura 3: Modelo final entre estilos de vida y autoconcepto mediante diagrama de ruta. Práctica: PCUI=Autocuidado y cuidado médico; PALI=H abitos alimenticios; PSPA=Consumo de alcohol, tabaco y otras drogas; PSU EÑ $0=$ Sueño; Creencia: $\mathrm{CO} C \mathrm{CIO}=$ Recreación y manejo del tiempo libre; $\mathrm{CCUI}=$ Autocuidado y cuidado médico; $\mathrm{CALI}=\mathrm{H}$ abitos alimenticios; $C S P A=$ Consumo de alcohol, tabaco y otras drogas. $A A=$ Autoconcepto Académico; $A S=A$ utoconcepto Social; $\mathrm{AF}=$ Autoconcepto Familiar; $\mathrm{AF}=$ Autoconcepto Físico.

\section{Discusión}

El presente trabajo permite identificar que el desa rrollo del autoconcepto no depende exclusivamente de los estilos de vida, aunque, un buen autoconcepto al parecer si impactaría el desempeño académico y social de los estudiantes universitarios, es más, se ha considerado al autoconcepto como un predictor de salud mental (M ontoya et al., 2013). En la literatura científica se ha reportado que los estudiantes universitarios colombianos tienen estilos de vida que ponen en riesgo su salud, como el sedentarismo, mala alimentación, y consumo de drogas, sin embargo, los estudios indican que los universitarios no identifica el riesgo de estas conductas, lo quese conoce como sensación de invul nerabilidad (Bastías y Stiepovich, 2014), además se ha identificado que las conductas de riesgo no solo afectan la sal ud general, tam- 
bién pueden tener un impacto negativo sobre el desempeño académico de los estudiantes (Alhomaid et al., 2017).

Por otra parte, un factor que se ha encontrado relacionado con los estilos de vida en población universita ria es su capital social, lo que indicaría que las relaciones sociales que establecen los jóvenes juegan un factor predictor de las conductas saludables (Mehri et al., 2016). I gualmente, los estilos de vida pocos saludables en universitarios también se pueden explicar por factores multidimensionales causados por fenómenos como la urbanización y la fal ta de acciones en promoción de la salud (Ramírez et al., 2015). Incluso, la forma como se maneja la información sobre los estilos de vida influye en la adopción de estos,Yang et al. (2017) realizaron un estudio con 700 estudiantes universitarios y hallaron que quienes son críticos con la información que reciben sobre salud mediante los medios digitales presentan más conductas sal udables.

Si bien se ha descrito una relación entre los estilos de vida y el autoconcepto (Luquiens et al., 2016), la evidencia todavía no es concluyente con respecto a esta relación, por ejemplo, Frazier et al. (2015) en una investigación con una muestra de 1612 estudiantes universitarios se encontró que el hábito del tabaquismo no se asociaba al autoconcepto físico, mientras que, el sobrepeso si se asoció fuertemente, por otra parte, $N$ azar et al. (2019) reportaron que los hombres entre las edades de 22 a 24 años con un nivel de autoconcepto social alto tenían más probabilidades de pertenecer a grupos que exhibían estilos de vida de alto riesgo.

No obstante, Chacón-Cuberos et al. (2020) reportan un mayor autoconcepto físico, social y académico en quienes practican una actividad física, sin embargo, Bohórquez et al. (2019), mencionan que se requiere de más estudios que cuestionen si la ausencia de actividad física suponeun autoconcepto social diferente al de aquellos que practican esta actividad de manera individual 0 colectiva.

Ahora bien, un aspecto a tener en cuenta en los estudios realizados con universitarios, es que se ha evidenciado que la formación en educación superior implica tanto el aprendizaje de una disciplina, como la generación de aptitudes emocionales, personales y sociales positivas (M ontoya et al. , 2018), por lo tanto, en futuras investigaciones sería relevante explorar el peso quetiene la educación superior frente a otras variables como los hábitos de vida saludables, competencias ciudadanas, cognitivas y emocionales que podrían explicar la formación del autoconcepto.
Respecto a las limitaciones de la presente investiga ción, si bien se cuenta con una muestra probabilística, los resultados únicamente se pueden generalizar a la institución universitaria de donde salieron los participantes, por lo tanto, sería pertinente contrastar los resultados del estudio con muestras tomadas de otras instituciones de educación superior; también, el estudio al ser de tipo transversal no aporta evidencia de los cambios en la formación del autoconcepto en el transcurso de la formación universitaria; por último, es urgente contar con estudios psicométricos y datos normativos para la cultura colombiana a nivel universitario, que permitan disminuir sesgos de evaluación para los dos cuestionarios implementados en el presente estudio, las anteriores tres limitaciones se sugieren en estudios posteriores.

\section{Agradecimientos}

Este artículo presenta los resultados de la investiga ción Factores asociados a los hábitos de vida saludable de los estudiantes de Psicología de la IUE, financiado por la 0 ficina de Investigaciones de la IUE, bajo la modalidad de Iniciativa Propia, en el año 2019.

\section{Referencias}

Aguirre-LoaizaH, Arenas],Ariasl, Franco-JímenezA, Barbosa GranadosS, Ramos-BermúdezS,AyalazuluagaF, Núñez C and García-Mas A (2019a). Effect of A cute Physical Exercise on Executive Functions and Emotional Recognition: Analysis of M oderate to High Intensity in Young Adults. Frontiers in Psychology, 10:2774. doi: 10.3389/ fpsyg. 2019.02774

Aguirre-Loaiza, H., Parra, J., Bartolo, L. J., Cardona, M. A. y Arenas, J. A. (2019b). Desempeño neuropsicológico e indicadores de frecuencia, duración y tiempo delasesión del ejercicio físico. Pensamiento Psicológico, 17(1), 19-32. doi:10.11144/ Javerianacali.PPSI17-1.dnif

Aldás, J. , \& U riel, E. (2017). A nálisisprevio de los datos. En Análisismultivariante aplicado con R (2nd ed., pp. 31-74). Madrid, España: Paraninfo.

Alhomaid,T.,Wadi, M.,Almanea, M.,AlTurki,A.,Alharbi, B., Aldubaykhi, M. , Alnashry, L., Alharbi, B., Alahmad, R., Alharbi, S. Alharbi, F. (2017). Lifestyle of M edical students đQ $\mathrm{Q}$ assim University. The Egyptian Journal of $\mathrm{H}$ ospital M edicine, 69 (7), 2918-2929. DO I: 10.12816/0042586

Alves, D. M., Almeida, L. M. y Fernandes, H. M. (2017). Estilos de vida e autoconceito: um estudo comparativo em adolescentes. Revista Iberoamericana de Psicología del Ejercicio y el D eporte, 12 (2), 237-247. 
Arántzazu, R., Goñi, A., \& Ruiz de Azúa, S. (2006). Autoconcepto físico y estilos de vidaen la adolescencia. Psychosocial Intervention, 15(1),81-94. Disponible: h t t p s : / / w w w. redalyc.org / articulo. oa?id $=179814011006$

Arrivillaga, M., Sal azar, I. y Gómez, I. (2002). Prácticas, creencias y factores del contexto relacionados con estilos de vida de jóvenes y adultos. D ocumento deTrabajo. Cali: Pontificia Universidad Javeriana.

BarbosaGranados, S. H. yA guirre-Loaiza, H. (2020). Actividad física y cal idad de vidarelacionada con lasalud en una comunidad académica. Pensamiento Psicológico, 18(2), 129. doi:https:/ / doi.org/ 10.11144/ Javerianacali.PPSI18-2.afcV

Bastías, E. \& Stiepovich, J. (2014). Unarevisión delosestilos de vida de estudiantes universitarios Iberoamericanos. Ciencia y enfermería, 20(2), 93101. https:// dx.doi.org/ 10.4067/ S071795532014000200010

Bohórquez, M., Checa I. \& Ramis, Y. (2019). Estudio de invarianciadel autoconcepto social en practicantes deactividad física individual y colectiva. Revista dePsicol ogía del Deporte28(1), 125-130.

Byrne, B. (2001). Structural equation modeling with AM OS. Basic concepts applica-tions, and programming. M ahwah, NJ: LEA.

Cachón-Zagalaz, J., Cuervo-Tuero, C., Zagalaz-Sánchez, M. L., y González-González de M esa, C. (2015). Relación entre la práctica deportiva y las dimensiones del autoconcepto en función del género y la especialidad que cursan losestudiantes delosgrados demagisterio. Journal of Sport and Health Research, 7(3), 257-266. https:/ / www. cabdirect. org/ cabdirect/ abstract/ 20153319964

Cardenal, V. y Fierro, A. (2003). Componentes y correlatos del autoconcepto en laescal a de Piers-H arris. Estudiosde Psicología, 24, 101-111. https:/ / www. uma. es/ Psicologia/docs/ eudemon/ investigacion/ componentes\%20y\%20correlatos\%20de\%20autoconcepto.pdf

Carmona Rodríguez, C. y Sánchez D el gado, P. y Bakieva, M . (2011). Actividades Extraescolaresy Rendimiento Académico: Diferencias en Autoconcepto y Género. Revista delnvestigación Educativa, 29(2), 447-465.

Cervantes, J., delToroValencia, M. y Chávez López. J. (2018). Estilos de vidaen estudiantes del áreade lasalud. Integración Académica en Psicología, 6(17), 50-58. http:/ / www. alfepsi. org/ wp-content/ uploads/ 2018/ 05/ Integracion-Academica-en-Psicologia-V6N 17. pdf

Chacón-Cuberos, R., Zuritz-O rtega, F., García-Marmol, E., \& Castro-Sánchez, M. (2020). Autoconcepto multidimensional según prácticadeportivaen estudiantes universitarios de Educación Física de Andalucía. Retos, 37(37), 174-180. Doi: https:/ / doi.org/ 10.47197/ retos. v37i37.71861
Colpsic. (2012). D eontología y bioética del ejercicio dela psicología en Colombia. Bogotá, Colombia: Offsetgraf.

Esteves, D., Vieira, S., Brás, R. , O'Hara, K. y Pinheiro, P. (2017). Nível de atividade física e hábitos de vida saudável de universitários por tugueses. Revista Iberoamericana de Psicología del Ejercicio y el Deporte, 12(2),261-270. https:/ / www. redalyc. org/ articulo.oa?id=3111/ 311151242009

Frazier, L. D.,Vacarro, J.A., Garcia, S., Fallahazad, N., Rathi, K., Shrestha, A.\& Perez, N. (2015) Diet self-efficacy and physical self-concept of college students at risk for eating disorders. I Behav H ealth, 4(4):97-100. doi:10.5455/ jbh.184462

García, F. y M usitu, G. (2001). Autoconcepto Forma 5.AF5. Manual. Madrid:TEA.

García, F. y M usitu, G. (2014). AF-5:Autoconcepto Forma 5. Ma drid, España:TeaEdiciones.

Gómez-A costa, C. (2018). Factorespsicológicospredictores deestilosdevidasaludable. RevistadeSalud Pública, 20(2), 155-162. https:/ / doi.org/ 10.15446/ rsap.v20n2.50676 Gómez-M azorra, M., Sánchez-O liva. , D. \& \& LabisaPalmeira, A. (2020). Actividad físicaen tiempo libreen estudiantes universitarioscolombianos. Retos, 37 (1), 181-189. doi: https: / / doi. org/ 10.47197/ retos. v37i37.71495

Guedeaz Delgado, J. C., Solano-Pinto, N., Blanco-O rnelas, J. R., Ceballos-Gurrola, 0., \& Zueck-Enríquez, M. C. (2017).Autoconcepto físico, género y cuidado delasal ud en universitarios mexicanos. Revista de Psicología Del De porte, 26, 51-59.

Hair, J. F., Hult, G. T., Ringle, C. M. , \& Sarstedt, M. (2017). A Primer on Partial Least Squares Structural Equation M odeling (PLS-SEM) (2nd ed.). U nited States of America: SAGE Publications Inc.

Harter, S. (1990). Issuesin the assessment of the self-concept of children and adolescents. En A.M. La Greca (Ed.), Through theeyes of thechild: Obtaining self-reportsfrom children and adolescents (pp. 292-325). Boston: Allyn \& Bacon.

Harter, S. (2012). Theconstruction of theself. D evelopmental and Sociocultural Foundations ( $2^{\text {nd ded }}$.). NewYork:The Guilford Press.

Herazo-Beltran,Y., Nuñez-Bravo, N. , Sánchez-Guette, L., Vásquez-O sorio, F., Lozano-Ariza, A., Torres-Herrera, E. yValdelamar-Villegas, A. (2020). Estilos de vidarelacionados con la salud en estudiantes universitarios. Retos, 38 (2), 547-551. https:/ / recyt. fecyt. es/ index. php/ retos/ article/ view/72871/ 0

Huebner, E. S., Valois, R., Suldo, S., Smith, Q., M cN ight, C., Seligson, J. yZullig, K. (2004). Perceived qual ity of life: A neglected component of adolescent health assessment and intervention. Journal of Adolescent $H$ ealth, 34, 270-278. https:/ / doi.org/ 10.1016/ j.jadohealth. 2003.07.007

Jöreskog, K. G. (1977). Structural Equation M odels in the Social Sciences: Specification estimation and testing $\ln P$. 
R. Krishnaiah (Ed.), Applications of Statistics (pp. 265-287). Amsterdam: North Holland Publishing Company.

López-Barajas, D., O rtega-Álvarez, F., Valiente-Martínez, I., y Zagalaz-Sánchez, M. (2010). Estudio comparativo del autoconcepto físico en adolescentes en función del género y del nivel de actividad físico-deportiva. Retos. N uevas tendenciasen Educación Física, Deportey Recreación, 17, 3841. https: / / www. redalyc. org/ articul 0. oa?id=3457/ 345732283008

Luquiens, A, Falissard B. \&Aubin, H.J. (2016). Studentsworry about the impact of alcohol on quality of life: roles of frequency of binge drinking and drinker selfconcept. DrugAlcohol Depend, 167, 42-8. doi:10.1016/ j.drugalcdep. 2016.07.031

Marsh, H.W. (2001, M ayo). A multidimensionalphysical selfconcept: a construct validity approach to theory, measurement and research. Comunicación presentadaal 10thW orld Congress of Sport Psychology, G reece.

Mehri,A., Solhi, M., Garmaroudi, G., Nadrian, H. . \& Shahbazi, S. (2016). Health promoting lifestyleand itsdeterminants among university studentsin Sabzevar, Iran. Int J Prev M ed, 7 (65). Doi: 10.4103/ 2008-7802.180411

Montoya, D. M. , Dussán, L. C., Hernández B. S., y delaRosa, J. (2013). Autoconcepto en una muestra de estudiantes universitarios de pregrado en medicina de la ciudad de Manizales (Colombia). Archivos de M edicina (Col), 15(1), 57-66. https:/ / www. redalyc. org/ pdf/ 2738/ 273840435006.pdf

M ontoya, D., Pinilla,V., \& Dussán C. (2018). Caracterización del autoconcepto en unamuestradeestudiantesuniversitarios de al gunos programas de pregrado de laciudad de M anizales. Psicogente, 21(39), 162-182. https:/ / dx.doi.org/ 10.17081/ psico.21.39.2829

Nazar, G., Stiepovic, J., \& Bustos, C. (2019). Latent class analysis of lifestyle behavior among Chilean university students. International J ournal of Health Promotion \& Education, 57(2), 98111. https:/ / doi-org. iue. basesdedatosezproxy.com/ 10.1080/ 14635240.2018.1552534

O Imedilla, A., O rtegaT. E., yA benza, L. (2016). Self-concept, sport, and physical activity practicein university students. Journal of Human Sport and Exercise, 11(4), 415-425. doi:10.14198/ jhse.2016.114.02

O netti-O netti,W., Chinchilla-M inguet, J. L., M artinsF. M. L. yCastillo-Rodríguez,A. (2019). Self-Conceptand Physical Activity: DifferencesBetween High School and U niversity Students in Spain and Portugal. Front Psychol., 10, 1333. doi: 10.3389/ fpsyg 2019.01333

O rganización M undial delaSalud (O MS, 2004). Promoción de la salud. Conceptos. Evidencia Emergente. Práctica. Informe Compendiado. https:/ / www.who.int/ mental_health/ evidence/ promocion_de_la_salud_mental.podf
Páez, M . L. \& Castaño, J. J. (2010). Estilos de viday salud en estudiantes de unafacultad de psicología. Psicología desde el Caribe, 25, 155-178. https:/ / www. redalyc. org/ articulo. oa?id=213/ 21315106008

Pinilla-Sepúlveda, V. E., M ontoya-Londoño, D. M ., DussánLubert, C. y Hernández-Botero,J. S. (2014).Autoconcepto en unamuestrade estudiantesuniversitarios delaciudad deM anizales. Revista H acia la Promoción dela Salud, 19(1), 114-127. https: / www. redalyc.org/ articulo. oa?id=3091/ 309131703009

Ramírez, R. et al (2015). A cross-sectional study of Colombian University students' self-perceived lifestyle. SpringerPlus 4 (289), 1-8. https:/ / doi. org/ 10.1186/ s40064-0151043-2

Resolución 8430 de 1993 del M inisterio de Salud y Protección Social. Por la cual se establecen las normas científicas, técnicasy administrativas para la investigación en salud. Colombia, 4 deoctubre de 1993.

Rivero-Rodríguez, G. (2011). Análisis de datosincompletos en CienciasSociales. M adrid, España: Centro deInvestigacionesSociológicas.

Rodríguez-Espinosa, H. , Restrepo-Betancur, L. F. \& DeossaRestrepo, G. C. (2015). Conocimientosy prácticas sobre alimentación, salud y ejercicio en universitarios de Medellín-Colombia. Perspectivas en Nutrición Humana, 17 (1), 36-54. https:/ / dx.doi.org/ 10.17533/ udea. penh.v17n1204

Ross-H oule, K., \& Q uigg, Z. (2019). Content, perceptions and impact of alcoholic drink promotions in nightlife venues that are targeted towards students. Addictive Behaviors Reports, 9(100163), doi: https:/ / doi.org/ 10.1016/ j.abrep. 2019.100163

Sánchez-O jeda, M. y deLuna-Bertos, E. (2015). Hábitos de vida saludableen la población universitaria. N utrición H ospitalaria, 31(5), 1910-1919.

Veliz-Burgos, A. y U rquijo, P. A. (2012). Niveles de autoconcepto, autoeficaciaacadémicay bienestar psicológico en estudiantes universitarios de laciudad deTemuco. Salud \& Sociedad: investigaciones en psicología de la salud y psicología social, 3(2), 131-150. http:// p e p s i c. b v s a l ud.org / sciel 0. php?script $=$ sci_arttext $\&$ pid $=$ S0718$74752012000200002 \& \operatorname{lng}=$ pt\&tlng $=e s$.

Xenakis, N. y Goldberg, J. (2010). The Young Women's Program: A health and wellness model to empower adolescentswith physical disabilities. Disability and $H$ ealth Journal, 3(2), 125-129. doi: 10.1016/ j.dhjo.2009.08.001

Yang, S. C. , Luo,Y. F. \& Chiang, C. H. (2017). TheAssociations Among Individual Factors, eH ealth Literacy, and $\mathrm{H}$ ealthPromoting Lifestyles Among College Students J. M ed Internet Res;19 (1): el5. Doi :10.2196/ jmir.5964 